Postoperative outcomes of cytoreductive surgery(CRS) with

hyperthermic intraperitoneal chemotherapy(HIPEC)

Yasuma F, Sekiguchi S, Takenouchi K, Noma S, Maehara Y

Dept. of Anesthesiology, National Center for Global Health and Medicine

\section{BACKGROUND}

CRS with HIPEC is performed to treat diseases such as pseudomyxoma peritonei(PMP), peritoneal carcinomatosis (PC), and malignant mesothelioma. It's highly invasive, and can result in various complications. This study retrospectively examined the postoperative outcomes and factors contributing to the outcomes in our hospital.

CRS- the complete resection of any visible peritoneal tumor HIPEC- to remove microscopic peritoneal disease by delivering heated chemotherapy drugs to the abdominal cavity

- the open abdomen technique was used - for $30-60 \mathrm{~min}, 41^{\circ} \mathrm{C}$

\section{METHODS}

Subjects were 166 patients who underwent this surgery between October 2010 and October 2017. Data were extracted from the anesthesia information management system and electronic health records in our hospital. The relationships between postoperative outcomes and patient backgrounds or intraoperative factors were retrospectively examined.

$>$ Statistical analysis was performed using nonparametric tests. Descriptive statistics are presented as median and ranges for continuous variables and as counts for categorical variables. All analyses were performed with $J M P \circledast(13.0 .0)(P<0.05)$.

\title{
RESULTS and DISCCUSIONS
}

Table I: Patient background $n=166$

\section{Age; years}

Gender; Male: Female

Body Mass Index; $\mathrm{kg} \mathrm{m}$

ASA class I / II / III (n)

Diagnosis ( $\mathrm{n}$ )

PMP

118

PC

Mesothelioma etc.

Peritoneal Cancer Index; score 42

6

$23(I-39)$

\section{Table2. Intraoperative fluid balance}

\begin{tabular}{|l|l|c|c|}
\hline Input & Crystalloids; $\mathrm{mL}$ & $\mathbf{1 6 6}(100 \%)$ & $6800(2650-27200)$ \\
\hline & Colloids; $\mathrm{mL}$ & $146(88 \%)$ & $1500(200-7750)$ \\
\hline 5\%alubmine; $\mathrm{mL}$ & $141(85 \%)$ & $1000(250-4000)$ \\
\hline RBC; $\mathrm{mL}$ & $144(87 \%)$ & $1120(280-6160)$ \\
\hline FFP; $\mathrm{mL}$ & $163(98 \%)$ & $1860(450-6330)$ \\
\hline & Platelets; $\mathrm{mL}$ & $23(14 \%)$ & $250(100-1000)$ \\
\hline Output & Blood loss; $\mathrm{mL}$ & 166 & $627(25-26050)$ \\
\hline & Urine; $\mathrm{mL}$ & 166 & $1005(160-13270)$ \\
\hline & Ascites; $\mathrm{mL}$ & 153 & $2369(25-15644)$ \\
\hline
\end{tabular}

Table3: Perioperative parameters

Anaesthesia time; minutes

$791(454-166 \mid)$

Operative time; minutes

$682(367-1526)$

Postoperative ventilation; days

$2(0-10)$

Length of ICU stay; days

$6(2-25)$

Hospital stay; days
Table4. Major Complications $n=39 / 166$ (23.5\%)

\begin{tabular}{|l|c|}
\hline Intra-abdominal bleeding & II (6.6\%) \\
\hline Respiratory complications & $6(3.6 \%)$ \\
\hline Cardiovascular complications & $4(2.4 \%)$ \\
\hline Pancreatic fistula & $4(2.4 \%)$ \\
\hline DIC & $3(1.8 \%)$ \\
\hline Death & I (0.6\%) \\
\hline Others (stroke, septic shock, etc.) & 14 \\
\hline
\end{tabular}
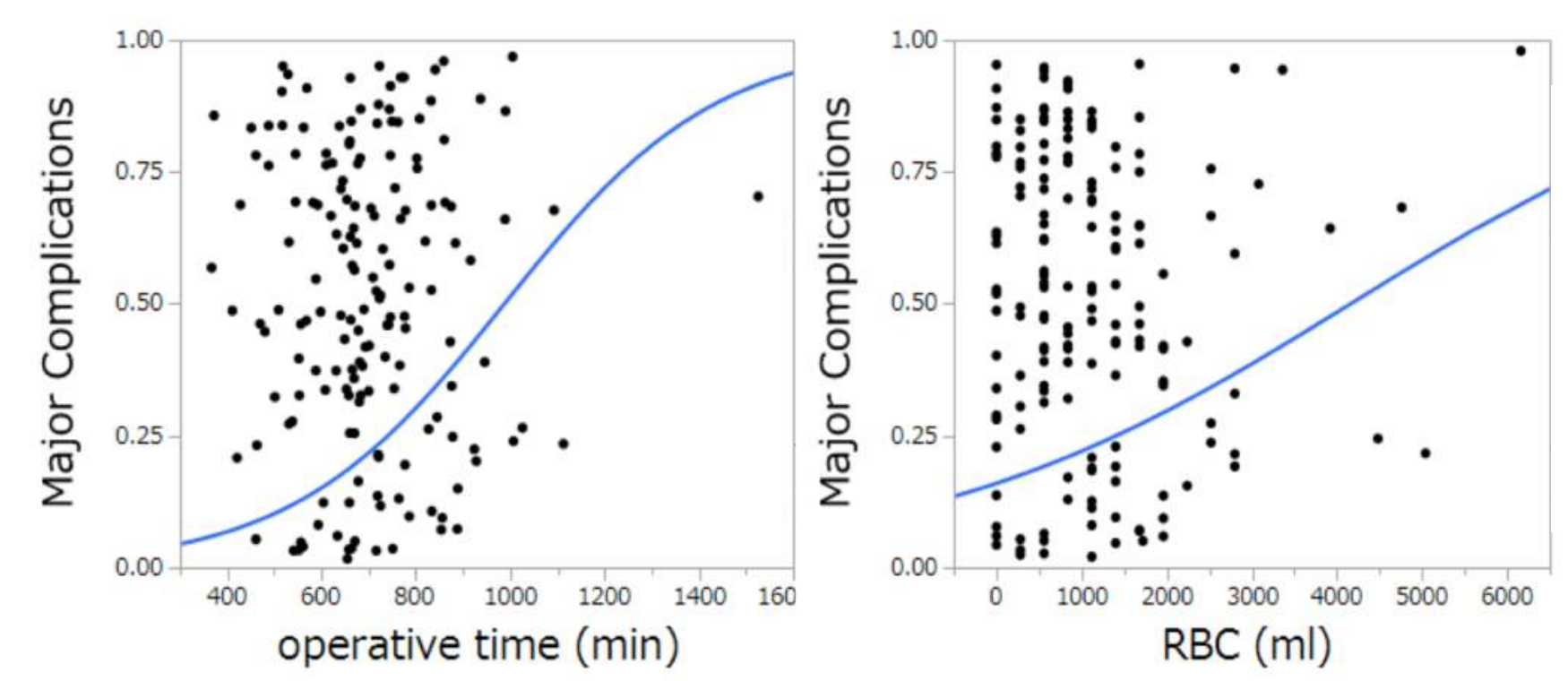

> Risk factors for all complications were operative time, crystalloid infusion, and red blood cell transfusion. The greatest risk factor was operative time, which was considered in relation to the number of resected organs, extent of lesions, and adhesiolysis.

$>$ Previous studies have also reported that complications of the surgery were associated with operative time, transfusion volume, $\mathrm{PCl}$, diaphragmatic peritonectomy, and number of resected organs.

\section{CONCLUSION}

